\title{
Abhandlungen
}

\author{
Udo E. Simonis
}

\section{Strukturwandel der Wirtschaft und Entlastung der Umwelt}

\begin{abstract}
Schrumpfen und Wachsen sind, wie die theoretische Reflexion zeigt, inhärente Bestandteile des Strukturwandels der Wirtschaft und sie sollten, wie die empirische Analyse erweist zu elementaren Bestandteilen einer ökologischen Strukturpolitik werden. In diesem Aufsatz wird anhand vergleichender empirischer Studien festgestellt dass der autonome wirtschaftliche Strukturwandel zwar eine notwendige, jedoch keine hinreichende Bedingung einer nachhaltigen Entwicklung (sustainable development) ist. In der Praxis ist Politik daher weiterhin gefragt - eine effektive ökologische Politik allerdings, die vor allem - aber nicht ausschließlich - von zwei Ressorts formuliert und implementiert werden muss: der Umwelt und der Wirtschaftspolitik,
\end{abstract}

\section{Nachhaltige Entwicklung: Der Notwendige Strukturwandel}

Im Bericht der Weltkommission für Umwelt und Entwicklung von 1987, der zu einer weltweiten Diskussion über nachhaltige Entwicklung (sustainable development) geführt hat finden sich zwei Definitionen dieses Konzepts: ,Entwicklung nachhaltig zu machen heißt dass die gegenwärtige Generation ihre Bedürfnisse befriedigt, ohne die Fähigkeit der zukünftigen Generationen zu gefährden, ihre eigenen Bedürfnisse befriedigen zu können“ (eigene Übersetzung und Hervorhebung).

Die damit angesprochene Frage der intergenerationellen Gerechtigkeit durchzieht alle Diskussionen um das Konzept und ist Bestandteil aller seither vereinbarten internationalen Abkommen zum Schutz der Umwelt geworden. Die andere Definition ist weniger bekannt für das hier zu behandelnde Thema jedoch von besonderer Bedeutung: „Eine nachhaltige Entwicklung ist ein Prozess der Veränderung in dem die Nutzung der Ressourcen, die Struktur der Investitionen, die Orientierung des technischen Fortschritts und die institutionellen Strukturen konsistent gemacht werden mit den zukünftigen und den gegenwärtigen Bedürfnissen“ (eigene Übersetzung und Hervorhebung).

Es gibt starke Vermutungen und zahlreiche Belege dafür, dass diese Bedingungen der Nachhaltigkeit beim vorherrschenden ,industriewirtschaftlichen Weltmodell“" nicht gegeben sind. In den letzten hundert Jahren hat sich die Weltbevölkerung mehr als verdreifacht auf zur Zeit 6,1 Milliarden Menschen. Nach den jüngsten Projektionen der Vereinten Nationen wird sich die Zahl der Weltbevölkerung im Jahre 2050 zwischen 7,3 (niedrige 
Variante) und 10,7 Milliarden (hohe Variante) bewegen - ein Wachstum der grundlegenden, Art. Wenn die materiellen Ansprüche dieser künftigen Generationen im Durchschnitt so hoch wie die der derzeit lebenden Generationen sein sollten, müsste die Erde also eine erhebliche Zunahme der Produktion und des Konsums verkraften bei weiterem Wirtschaftswachstum entsprechend mehr. Kann die Erde das verkraften, oder sind doch Schrumpfungen angesagt?

Herman E. Daly hat das potentielle Zukunftsdilemma mit einer einfachen, aber tiefsinnigen Metapher beschreiben, dem Übergang aus der „leeren“ in die „volle“ Welt (siehe Abbildung 1) : Die Wirtschaft als offenes Subsystem des globalen Ökosystems hat ständig expandiert und immer weitere ökologische Nischen besetzt. Die Frage allerdings, wie voll denn die immer voller werdende Welt schon sei, ist (bisher) nicht eindeutig zu beantworten.

\section{Abb. 1: Die Wirtschaft als offenes Subsystem des Ökosystems}

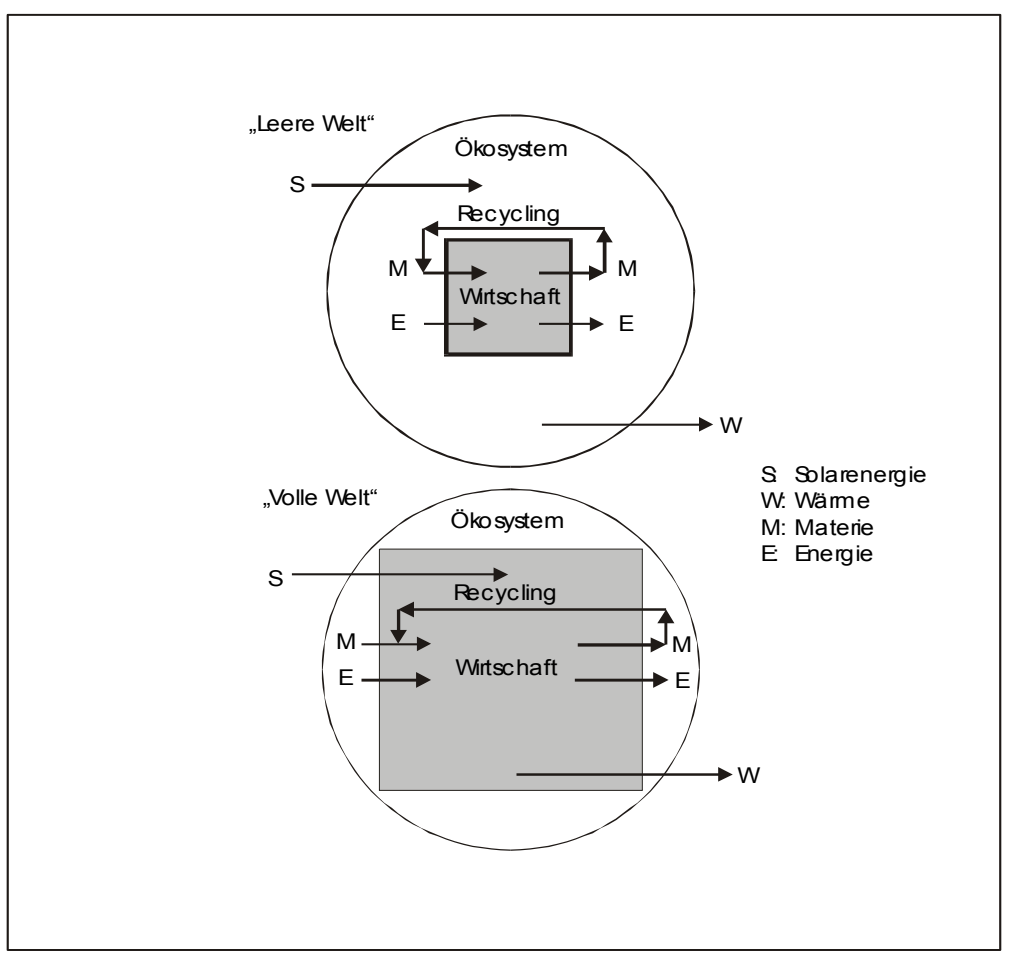

Quelle: Daly 1994, S. 148

Man muss die Frage der Zukunftsfähigkeit des industriewirtschaftlichen Weltmodells nicht in dieser theoretisch-abstrakten Weise stellen, um zu der Einsicht zu gelangen, dass tiefgreifende Veränderungen in der Art und Weise unseres Wirtschaftens erforderlich sind - und auch bevorstehen. Man kann dies auch anhand der drei ökologischen Managementregeln ableiten:

- Wird, erstens die Abbaurate des Verbrauchs nicht-erneuerbarer Ressourcen nicht drastisch gesenkt so wird es in gut einer Generation viele dieser Ressourcen nicht oder nur noch in begrenztem Umfang geben (z.B. Erdöl, tropischer Regenwald).

- Übersteigt, zweitens die Abbaurate erneuerbarer Ressourcen deren Regenerationsrate erheblich und über längere Zeit (wie das z.B. bei den Wasservorräten und Fischbeständen 
der Fall ist), dann kommt es zu Versorgungsengpässen und zum massiven Verlust der Artenvielfalt.

- Und wird, drittens, die Zuwachsrate der Belastung der Umwelt mit Schadstoffen nicht drastisch gesenkt - und in Minuswachstum verwandelt -, so wird die Absorptionskapazität der Natur nicht nur lokal und national (z.B. Boden- und Wasserkontamination), sondern auch global (z.B. Klimaänderung und Schädigung der Ozonschicht) überschritten.

So viel nur zu einer bedingten Status-quo-Prognose. Wie aber lassen sich, um das ja unmittelbar einleuchtende Gerechtigkeitspostulat im Bericht der Weltkommission von 1987 wieder aufzugreifen, zukünftige und gegenwärtige Bedürfnisse konsistent machen?

Ein Teil, der größte Teil einer Antwort auf diese Frage besteht wohl darin, dass die Wirtschaft systematisch durch umweltentlastenden Strukturwandel transformiert werden muss, dass es der Schrumpfung bei umweltzerstörenden und der Ausweitung umweltverträglicher Aktivitäten bedarf. Die Formeln der nachhaltigen Entwicklung (sustainable development) müssen mit dem politischen Imperativ verbunden werden, dass (relative wie absolute) Umweltentlastungen im weiteren Prozess der Ökonomisierung der Erde immer dringlicher werden und umfassender sein müssen, wenn ökologisch auch nur der Status quo gewahrt werden soll. Was lässt sich im Hinblick auf einen solch umfassenden Strukturwandel der Wirtschaft bisher konstatieren?

\section{$2 \quad$ Umweltbelastender oder -entlastender Strukturwandel?}

\subsection{Zum Strukturbegriff}

Aus wirtschaftsstatistischer Sicht umfasst der Begriff „Struktur“ die Aufteilung einer ökonomischen Gesamtgröße in mehrere Teilaggregate. Strukturwandel besagt also, dass sich die prozentualen Anteile bestimmter Teilaggregate am Gesamt verändern. Ein Strukturwandel der Wirtschaft kann sich belastend, entlastend oder neutral auf die Umweltbedingungen (die ökologische Situation) auswirken. Als umweltpolitisch relevante Strukturdimensionen gelten im allgemeinen Sektoren, Regionen und Betriebsgrößen.

Was den sektoralen Strukturwandel angeht sind zwei wichtige Struktureffekte zu unterscheiden:

- der intersektorale Strukturwandel im Sinne der sich vollziehenden Veränderungen des Anteils eines Sektors (einer Branche) an der gesamtwirtschaftlichen Produktion. Diese Veränderungen sind nicht umweltneutral, da die verschiedenen Sektoren höchst unterschiedliche Umweltverbräuche und -belastungen aufweisen. Umweltentlastung entsteht, wenn das Produktionsvolumen in den umweltbelastenden Sektoren (Branchen) absolut oder zumindest relativ zurückgeht. Von „ökologischen Gratiseffekten“” spricht man, wenn die Zuwachsrate der Sektoren (Branchen), von denen negative Effekte auf die Umwelt ausgehen, geringer ist als die des Bruttosozial- bzw. Bruttoinlandsprodukts (Entkopplung).

- der intrasektorale Strukturwandel im Sinne der Veränderungen, die sich innerhalb der Branchen, Betriebe und Unternehmen vollziehen. Hierzu zählen sowohl Veränderungen der Produkte als auch der Produktionsprozesse (der Technik). Von einer Verringerung des „ökologischen Rucksacks“ spricht man, wenn herkömmliche Produkte und Techniken durch umweltfreundlichere Varianten ersetzt, der Energie- und Materialverbauch redu- 
ziert und risikoreiche Vor- und Zwischenprodukte vermieden werden.

Der umweltrelevante Strukturwandel beschränkt sich jedoch nicht nur auf Veränderungen zwischen und innerhalb der Sektoren (Branchen) einer Volkswirtschaft. Auch die regionale (räumliche) Dimension kann von Bedeutung sein, insbesondere wenn Produktionen wegen unterschiedlicher Umweltschutzbestimmungen abwandern oder wenn umweltbelastende Produktionen ins Ausland (z.B. Entwicklungsländer) verlagert, Vorproduktionen von dort bezogen und Abfälle dorthin exportiert werden (externe Entropie). Bei räumlich divergierender Absorptionskapazität der Umwelt kann räumlicher Strukturwandel zu einer gesamträumlich bzw. global niedrigeren oder aber höheren Umweltbelastung führen. Von „Öko-Dumping“ spricht man, wenn bestehende Unterschiede in den Umweltschutzbestimmungen strategisch ausgenutzt werden und zu gesamträumlich (global) verstärkter Umweltbelastung führen.

Inwieweit der betriebsgrößenmäßige Strukturwandel umweltrelevant ist, ist erst ansatzweise untersucht worden. Einerseits mögen kleine und mittlere Unternehmen über die aktuellen Umweltschutzbestimmungen wie über die staatliche Förderung von Umweltschutzinvestitionen häufig nur unzureichend informiert sein. Andererseits können ihre Mitarbeiter ökologisch sensibler, ihre Produkte und Produktionsverfahren innovativer sein als die von Großunternehmen.

Was die Bestimmungsfaktoren des umweltrelevanten Strukturwandels der Wirtschaft angeht wird in der Regel zwischen drei zentralen Faktoren unterschieden: Nachfrageänderungen, technischer Fortschritt und politische Rahmenbedingungen.

- Nachfrageänderungen bestimmen vor allem den sektoralen Strukturwandel, wobei sowohl Änderungen der privaten Konsumstruktur, der öffentlichen Umweltschutzausgaben, der Exportstruktur als auch der Nachfrage nach Vorleistungen bedeutsam sein können.

- Veränderungen der Produktionstechnik prägen insbesondere den intrasektoralen Strukturwandel. Umweltentlastung kann aus der Substitution von Stoffen, vor allem aber aus der Senkung der Energie- und Materialintensität der Produktion entstehen (Prozessinnovation).

- Eine Umweltentlastung hängt darüber hinaus wesentlich von den gesamtwirtschaftlichen Rahmenbedingungen ab. Die Schonung der Ressourcen und der Erhalt der Ökosysteme erfordern insbesondere eine umweltgerechte Finanzreform, die aus vier Bausteinen bestehen sollte (SRU 1996): Abbau von Vergünstigungen mit ökologisch negativer Wirkung, Verstärkung bereits bestehender umweltpolitisch motivierter Vergünstigungen, Einbau von Anreizen zu umweltgerechtem Verhalten in bestehende Abgaben, Einführung neuer Umwelt(lenkungs)abgaben.

\subsection{Zur Umweltrelevanz des sektoralen Strukturwandels}

Die Zusammenhänge zwischen Strukturwandel der Wirtschaft und Belastung bzw. Entlastung der Umwelt lassen sich theoretisch gut beschreiben. Wie aber sieht es mit der empirischen Bestimmung, dem konkreten Nachweis dieser Zusammenhänge aus? Im letzten Jahrzehnt sind mehrere Studien zum umweltent- bzw. -belastenden Strukturwandel durchgeführt worden, die zwei unterschiedlichen Mustern folgen: Neben der Frage, welche Schadstoffemissionen mit dem Wachstum und dem Strukturwandel der Wirtschaft einhergehen output-orientierte Studien), interessierte die Frage, welche Änderungen ökologisch relevan- 
ter Energie- und Materialverbräuche zur Entlastung der Umwelt führen (inputorientierte-Studien). Die Ergebnisse dieser Studien schränken die zuvor oft gehörte These vom positiven Zusammenhang von Strukturwandel und Umweltentlastung deutlich ein. Folgende allgemeine Feststellungen lassen sich treffen:

- Was den groß-sektoralen Strukturwandel (zwischen Landwirtschaft Industrie und Dienstleistungen) angeht, ist der statistisch feststellbare Trend zur ,Dienstleistungsgesellschaft“ teilweise ein Anzeichen der Auslagerung produktionsbezogener Dienstleistungen, stellt also keine wirkliche Reduzierung des Verbrauchs natürlicher Ressourcen dar.

- Die feststellbaren Unterschiede in den Emissionskoeffizienten zwischen Dienstleistungssektor und Industriesektor verringern sich, wenn man die durch Vorleistungen verursachten Schadstoffemissionen (ökologischer Rucksack) mit in die Betrachtung einbezieht.

- Der Dienstleistungssektor expandiert, aber mit zunehmender Nutzung von Wohnraum, Verkehr und Tourismus - alles Leistungen, die in der Regel durch hohen Energieverbrauch, durch Lärmbelastung und zusätzlichen Flächenverbrauch gekennzeichnet sind.

- Der Rückgang des Anteils der Landwirtschaft am Bruttoinlandsprodukt geht einher mit einer weiteren Intensivierung der landwirtschaftlichen Anbautechnik und der chemisch-physikalischen Bodenbelastung.

Die Studien des DIW-, des HWWA-, des Ifo- und RWI-Instituts haben gezeigt, dass ein umweltentlastender intersektoraler Strukturwandel in Deutschland in der Vergangenheit wenig ausgeprägt war. So hat das RWI-Institut beispielsweise (auf Basis aktueller Daten über die Emission von Luftschadstoffen, das Aufkommen von Produktionsabfällen und von Abwässern) 18 aus insgesamt 58 Branchen der deutschen Industrie ermittelt, die als besonders umweltintensiv bezeichnet werden können (unter ihnen die Chemie, die Mineralölverarbeitung, Steine und Erden, Eisen und Stahl, Holzbe- und -verarbeitung, Zellstoff-, Papier- und Pappeerzeugung). Zwar ist die gesamtwirtschaftliche Bedeutung dieser umweltintensiven Branchen, gemessen am Anteil der Beschäftigten, gesunken. Gemessen am Rückgang der Schadstoffemissionen ist der interindustrielle Strukturwandel aber eher als gering einzustufen. (Im Umkehrschluss bedeutet dies, dass dem intrasektoralen Strukturwandel die größere umweltentlastende Bedeutung zukommt.)

Vor diesem Hintergrund ist zu vermuten, dass solange die natürliche Umwelt in ihrer Nutzung und Belastung ökonomisch unterbewertet bleibt, immer auch Prozesse eintreten werden, die ein umweltbelastendes Wirtschaftswachstum begünstigen. Die bisherigen Ergebnisse der empirischen Wirtschaftsforschung lassen für die Bundesrepublik Deutschland daher keinen grundsätzlichen Schluss auf eine Zielharmonie zwischen Strukturwandel und Umweltschutz zu.

Wie aber stellt sich das Bild bei einem internationalen Vergleich dar? Gibt es Vorreiter, gibt es Nachzügler auf dem Weg zu einem umweltentlastenden Strukturwandel oder gar Sitzenbleiber?

\subsection{Fallstudien über inter- und intrasektoralen Strukturwandel}

Die internationalen Wandlungsprofile der Wirtschaftsstruktur sind in mehreren Studien anhand eines Vergleichs ausgewählter Bereiche hoher struktureller Umweltbelastung untersucht worden (vgl. Jänicke u.a. 1988, 1989, 1993). Für 32 Industrieländer wurde aufgezeigt, wie sich neun umweltbelastende Wirtschaftsaktivitäten (die Produktion von Rohstahl, 
Aluminium, Zement, Pestizide, Düngemittel, Chlor, Papier/Pappe sowie die Stromerzeugung und der Gütertransport), die für fünf besonders belastungsintensive Branchen (Eisenschaffende Industrie, NE-Metallerzeugung, Steine/Erden, Chemie, Papier- und Pappeherstellung) stehen, über einen Zeitraum von 20 Jahren (1970-1990) entwickelt haben. Dabei ging es nicht - wie in den anderen erwähnten Studien -um die dabei entstehenden Schadstoffemissionen, sondern um den Output der untersuchten Branchen in physischen Einheiten (in Tonnen, Kilowattstunden und Tonnenkilometern).

In vier vertiefenden Fallstudien (über Deutschland, Japan, Schweden und Portugal) wurde danach gefragt, welche ökologischen Wirkungen der Bedeutungsverlust (die Schrumpfung) von Branchen mit hoher Umweltbelastung hatte, und wie der Saldo der Branchenverschiebungen sich auswirkte (intersektoraler Wandel). Darüber hinaus wurden die Veränderungen innerhalb der Branchen untersucht (intrasektoraler Wandel) und zwar anhand des ökologisch sensiblen Verbrauchs an Energie, Wasser, Flächen sowie Gütertransportleistung und Abfallaufkommen. Die Ergebnisse der Studien können, stark verkürzt - wie folgt zusammengefasst werden (im Detail hierzu Jänicke u.a., 1993, 48-49; 142-154):

Was den intersektoralen Strukturwandel angeht lassen sich die 32 untersuchten Industrieländer in zwei große Gruppen einteilen: Länder mit „teilweise umweltentlastendem Strukturwandel“ (wie u.a. Deutschland, Frankreich, Japan, Schweden) und Länder mit „,durchgängig ungünstiger Strukturentwicklung“ (wie u.a. Portugal, Slowakei, Tschechien, Türkei).

Abb. 2: Wirtschaftlicher Strukturwandel in Frankreich 1970 bis 1990*

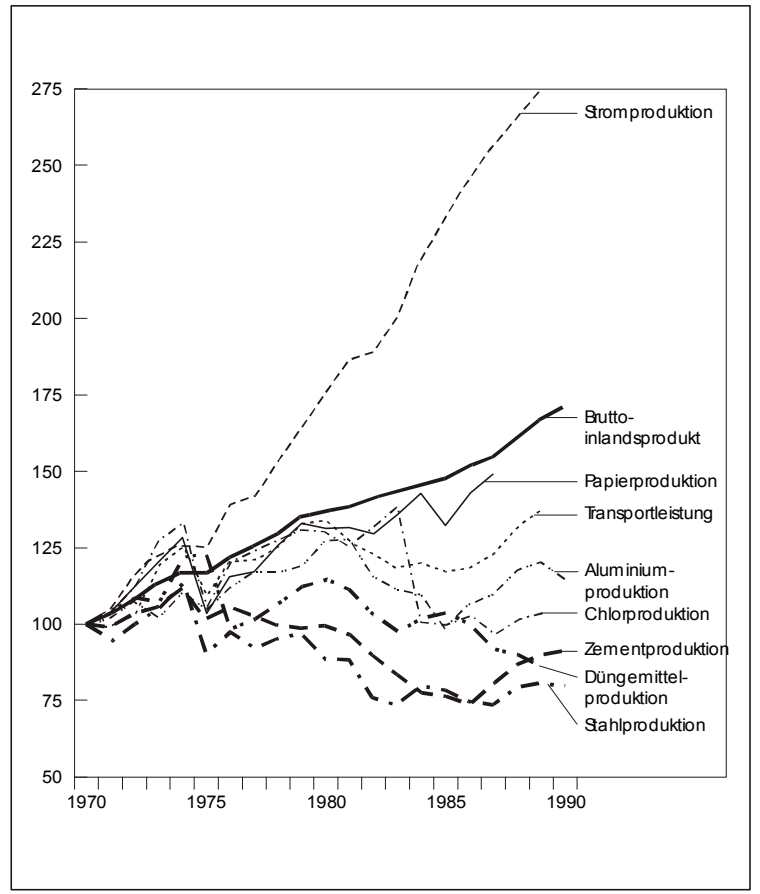

Quelle: Jänicke u.a. 1993, S. 62

$* 1970=100$ 
Das geschlossenste Bild der Entkopplung zwischen Bruttoinlandsprodukt und den ausgewählten neun umweltbelastenden Wirtschaftsaktivitäten im Zeitablauf ergab sich für Frankreich (vgl. Abbildung 2). Hier gingen die Zement-, die Dünger- und die Rohstahlproduktion absolut gesehen zurück und der Gütertransport stagnierte über längere Zeit; dagegen wies die Stromproduktion, insbesondere der Atomstrom, ein überproportionales Wachstum auf.

Während die Wachstumsraten von fünf der neun umweltbelastenden Wirtschaftsaktivitäten in Deutschland durchweg über dem Wachstum des Bruttoinlandsprodukts lagen, waren die Wachstumsraten in Japan und Schweden mehrheitlich niedriger.

Fasst man die ökonomisch am höchsten entwickelten Industrieländer als Gruppe zusammen, so lassen sich vier typische Wandlungsmuster ausmachen (vgl. Tab. 1).

Tab. 1: Wandlungsmuster in den hochentwickelten Industrieländern

\begin{tabular}{|llll|}
\hline $\begin{array}{l}\text { Absolute } \\
\text { Entlastung }\end{array}$ & $\begin{array}{l}\text { Relative } \\
\text { Entlastung }\end{array}$ & $\begin{array}{l}\text { Teils Schrumpfung/ } \\
\text { teils Wachstum }\end{array}$ & Anstiegsmuster \\
\hline $\begin{array}{l}\text { Zement } \\
\text { Stahl }\end{array}$ & $\begin{array}{l}\text { Energie* } \\
\text { Düngemittel } \\
\text { Transportgewicht }\end{array}$ & $\begin{array}{l}\text { Chlor* } \\
\text { Pestizide }\end{array}$ & $\begin{array}{l}\text { Elektrizität* } \\
\text { Straßengüter } \\
\text { verkehr } \\
\text { Papier* } \\
\end{array}$ \\
& Aluminium* \\
\hline * in Billigstromländern Belastungszunahme auf hohem Niveau & \\
\hline
\end{tabular}

Quelle: nach Jänicke u.a. 1993, S.49

Die mit Abstand negativste Strukturentwicklung ergab sich für die Türkei (vgl. Abbildung 3). Hier wurde das vergleichsweise hohe Wachstum des Bruttoinlandsprodukts durch überproportional hohe Zuwachsraten bei sieben der neun umweltbelastenden Wirtschaftsaktivitäten weit übertroffen. Ähnlich ungünstig verlief die Strukturentwicklung in Portugal.

Es lässt, sich also sagen, dass sich seit den 70er Jahren in den ökonomisch hochentwickelten Industrieländern durch Veränderungen in traditionellen Branchen wie Zement, Stahl und Düngemittel eine relative Umweltentlastung ergeben hat, in Form der Entkopplung (de-coupling) dieser Produktionen vom Bruttoinlandsprodukt; eine tendenzielle Entkopplung ergab sich auch beim Energieverbrauch. Dagegen wiesen die ökonomisch weniger entwickelten Industrieländer in den genannten Sektoren absolut zunehmende Belastungen auf, am stärksten ausgeprägt in Südosteuropa. Was die Stromproduktion und den Straßengüterverkehr betrifft, kam es auch in den hochentwickelten Industrieländern zu keiner Entkopplung vom Bruttoinlandsprodukt sondern zu einem weiteren Zuwachs (re-coupling) auf bereits hohem Niveau. Für eine Reihe von Industrieländern gilt dies auch für die Branchen Aluminium, Chlor, Papier/Pappe und Pestizide. 
Abb.3: Wirtschaftlicher Strukturwandel in der Türkei 1970 bis 1990*

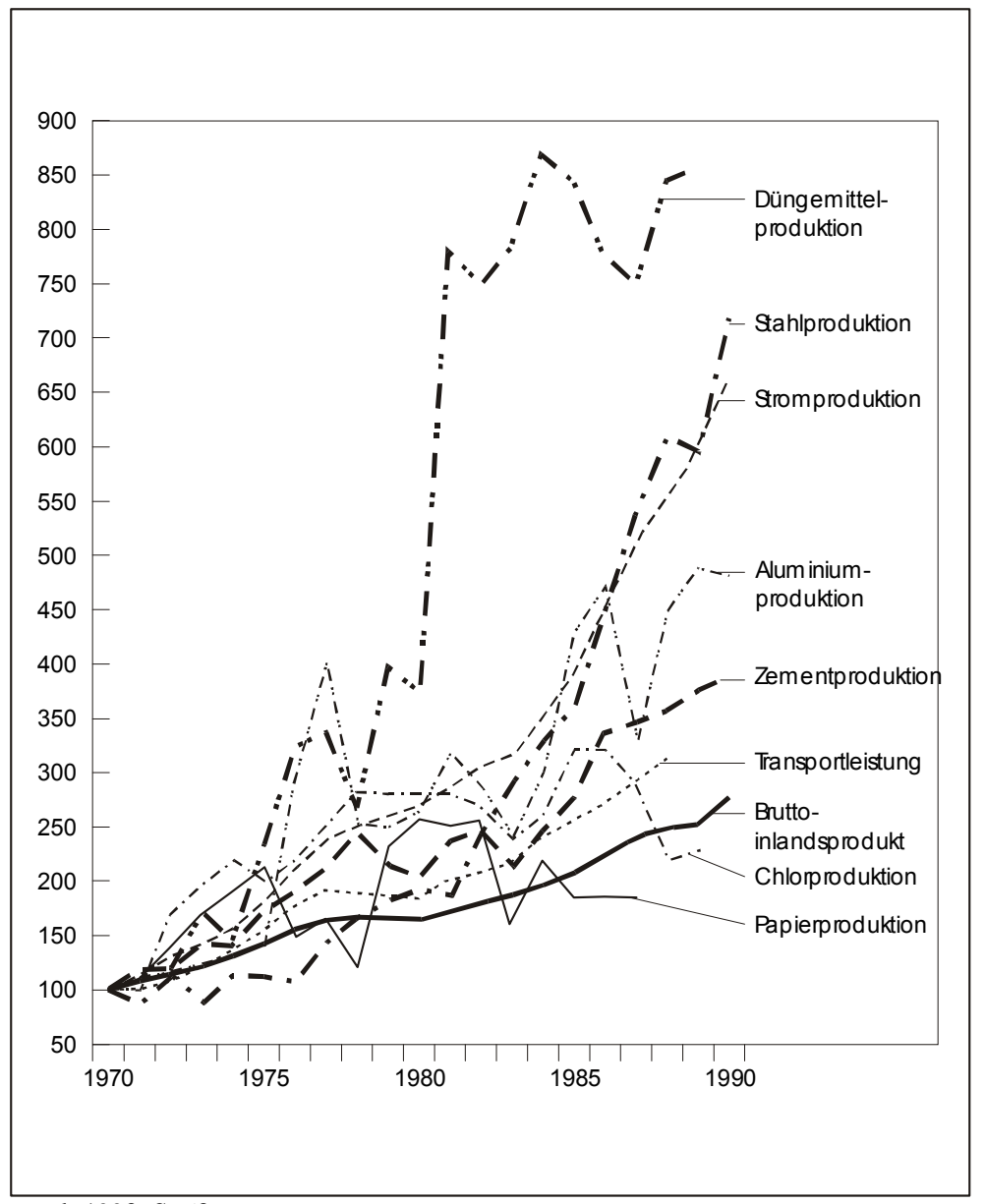

Quelle: Jänicke et al. 1993, S. 63

$* 1970=100$

Was den intrasektoralen Strukturwandel angeht, kommen auch Jänicke u.a. zu dem Schluss, dass ihm wohl größere Bedeutung zukommt als dem intersektoralen Wandel. Am stärksten ausgeprägt war die Entlastung beim industriellen Endenergieverbrauch; in Japan, Schweden und Deutschland ging er absolut zurück. Ökologisch günstig entwickelte sich auch der industrielle Wasserverbrauch, der in diesen drei Ländern ebenfalls absolut zurückging. Die Entlastungs- bzw. Belastungstendenzen in Bezug auf fünf ökologisch relevante Kategorien sind in Tabelle 2 für vier Industrieländer dargestellt.

Zusammenfassend lässt sich festhalten, dass auch beim intrasektoralen Strukturwandel in den Industrieländern in den letzten Jahrzehnten per Saldo nicht von einer signifikanten Umweltentlastung gesprochen werden kann. Andererseits darf nicht verkannt werden, dass ohne den erfolgten Strukturwandel sich die Konflikte zwischen dem ökonomischen und dem ökologischen System ohne Zweifel weiter verschärft hätten. 


\section{Tab. 2: Intraindustrieller Strukturwandel in vier Ländern}

1) Abfallaufkommen: 1975 -1985; Wasserverbrauch seit 1980 konstant; Flächenverbrauch: 1973-1985; Rohstoffverbrauch 1970-1990

2) Abfallaufkommen: 1977-1987; Rohstoffverbrauch (wertmäßig): ab 1978

3) Wasserverbrauch: amtliche Schätzung

4) Wasserverbrauch: 1980-1984; Energieverbrauch: 1977-1987

$+=$ Anstieg der Intensität / - = Rückgang der Intensität

Quelle: nach Jänicke u.a. 1993, S.148

\section{Ein nicht hinreichender Strukturwandel: Der politische Handlungsbedarf}

Die theoretische Ableitung deutete es an, und die empirische Überprüfung hat es belegt: Der autonome wirtschaftliche Strukturwandel ist eine notwendige, nicht aber eine hinreichende Bedingung einer nachhaltigen Entwicklung. Wenn selbst in den hochentwickelten Industrieländern eine durchgängige Entkopplung von Wirtschaftswachstum und umweltbelastender Produktion von alleine nicht zustandekommt, wird eine starke Umweltpolitik erforderlich, die aus ökologischen Gründen nicht nur eine relative, sondern auch eine absolute Reduzierung des Ressourcenverbrauchs und der Umweltbelastung erbringen muss. In der (politisch relevanten) Umkehrform heißt dies: Die Wirtschaften der industrialisierten Länder müssen ihre Ressourcenproduktivität weiter drastisch verbessern, wenn sie ökologisch zukunftsfähig werden wollen. Über die Größenordnung, um die es dabei geht, entsteht zunehmend Klarheit und auch ein allmählich wachsender Konsens: Mittelfristig geht es um mindestens einen "Faktor 4", langfristig um einen "Faktor 10" (vgl. Tabelle 2).

In jüngster Zeit sind überzeugende Argumente vorgebracht worden, dass in den Industrieländern eine "Faktor 10"-Strategie formuliert und umgesetzt werden sollte. Eine drastische Dematerialisierung der industriellen Wirtschaft (um den Faktor 10) erscheint nicht nur aus ökologischen Gründen erforderlich, sondern auch aus ökonomischer Sicht vorteilhaft gegenüber einer Umweltpolitik, die nicht auf das (vorsorgliche) Ziel der Ressourcenproduktivität sondern auf das (nachsorgende) Mittel der Schadstoffbehandlung ausgerichtet ist. 


\section{Abb. 4: Mögliche Pfade des globalen Materialverbrauchs in vH}

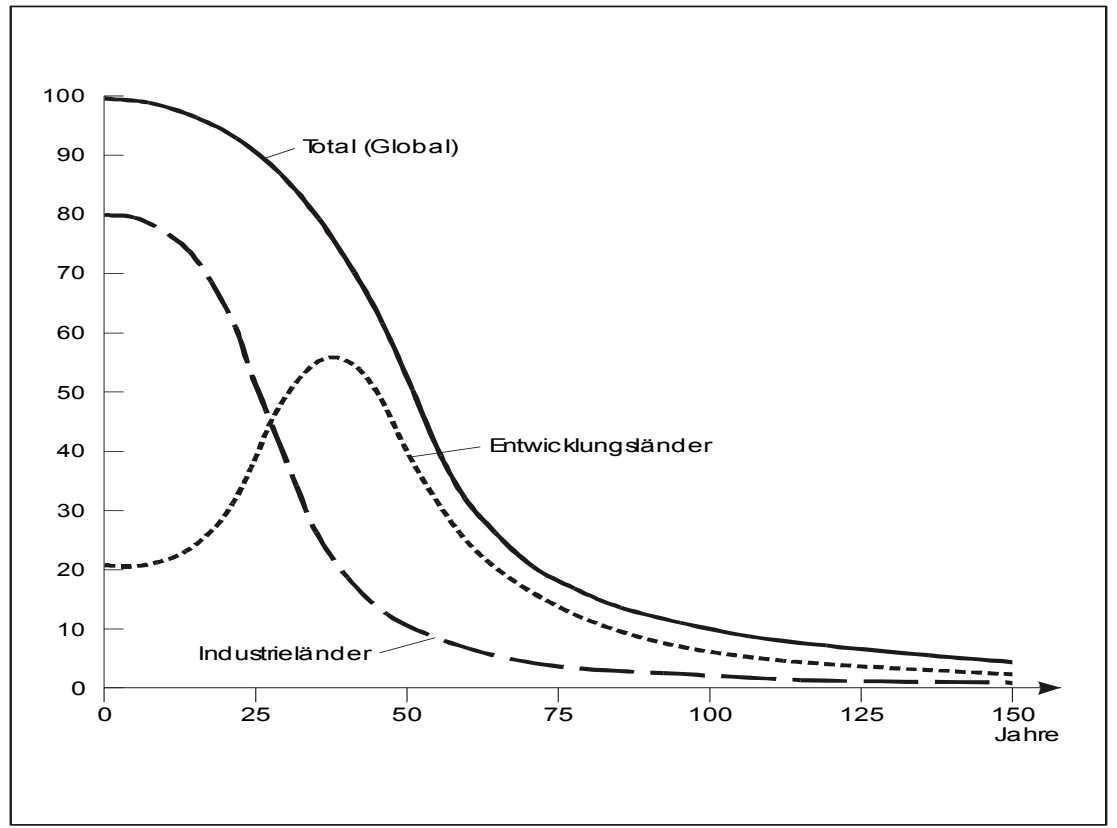

Quelle: Hinterberger u.a.1996, S. 86

Eine ökologisch begründete Schrumpfungsstrategie (Verringerung der Energie- und Materialströme der Wirtschaft) entspricht im konkreten Fall einer ökonomisch begründbaren Effizienzstrategie (Erhöhung der Ressourcenproduktivität). Eine sozial begründete Verteilungsstrategie (Verringerung der nationalen und globalen Ungleichheiten) kann einer ethisch begründbaren Suffizienzstrategie (gleiche Rechte bei der Nutzung und gleiche Pflichten beim Schutz der Natur) entsprechen.

Effizienz als traditionelles ökonomisches Prinzip und Suffizienz als zukünftig notwendiges soziales Prinzip müssen aber, das haben die zitierten Studien indirekt auch erbracht, ökologisch ergänzt werden durch eine Konsistenzstrategie (vgl. hierzu Abbildung 5). Konsistenz erfordert, auf das Thema Strukturwandel angewendet, die umweltverträgliche, qualitative Beschaffenheit von Stoff- und Energieströmen, das ökologisch begründete Inputmanagement der Produktion. Konsistente Stoff- und Energieströme sind solche, die entweder störsicher im geschlossenen Kreislauf geführt werden oder aber mit den Stoffwechselprozessen der Natur so weit übereinstimmen, dass sie sich, auch bei großem Volumen, problemlos darin einfügen. 


\section{Abb.5: Strategietypen zukunftsfähiger Entwicklung}

\section{Suffizienz}

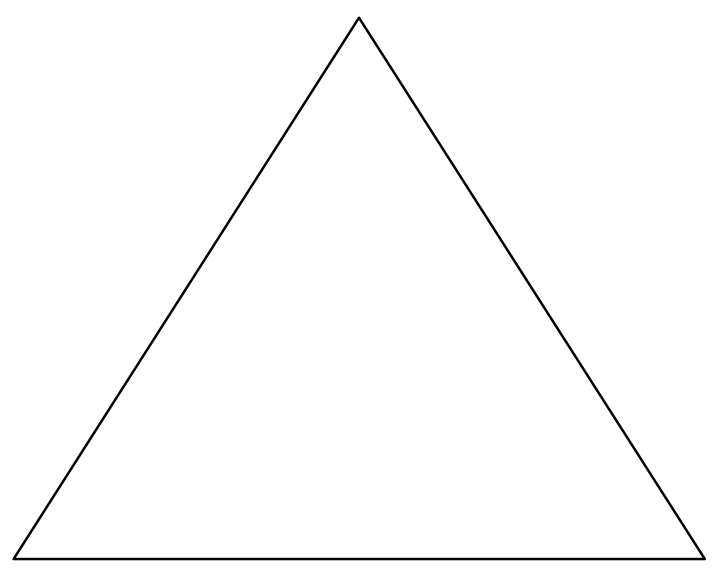

\section{Effizienz}

\section{Konsistenz}

Quelle: nach Huber 1995

Angesichts der eher bescheidenen Erfolge von Umweltentlastung durch Strukturwandel wird in Wirtschaft und Politik zunehmend mit dem Leitbild der „Kreislaufwirtschaft“ hantiert, wo es, wie die empirischen Studien belegen, eigentlich um „Stoffwechselwirtschaft“, um industriellen Metabolismus gehen müsste. Gerade weil der stoffliche und energetische Grundumsatz der ,industriewirtschaftlichen Weltmodells“ viel zu hoch liegt, ist dieses Modell ja nicht nachhaltig und auf die Welt als Ganzes nicht übertragbar. Das Modell muss also selbst erst einmal nachhaltig gemacht werden.

Der "materielle Kern" des weiterhin bestehenden Konflikts zwischen Ökonomie und Ökologie besteht darin, dass weder Umweltbelastung als Kostenfaktor noch Umweltentlastung als Erlösfaktor im Marktgeschehen systematisch Berücksichtigung finden. Die natürliche Umwelt wird daher übermäßig, nicht optimal genutzt. Der einzelne Produzent, das Unternehmen, der Sektor, die Region leistet keinen ausreichenden Beitrag zum Umweltschutz, wenn und solange sich dadurch die Kosten- und/oder Wettbewerbssituation verschlechtert. Der einzelne Konsument leistet freiwillig keinen ausreichenden Beitrag zum Umweltschutz, wenn er dafür mehr zahlen muss, ohne dass diesem "Opfer" ein spürbarer Nutzen gegenübersteht. Und der Staat leistet keinen überzeugenden Beitrag zum Umweltschutz, wenn und solange er an zunehmender Umweltbelastung verdient (Einnahmeseite des Budgets) oder wie sein Verhalten (Ausgabeseite des Budgets), zu weiterem Ressourcenverbrauch und zusätzlicher Umweltbelastung führt.

Diese elementaren, aber essentiellen Zusammenhänge bedeuten, dass es bei mikro-ökonomischem Rationalverhalten bisher keine hinreichenden Anreize gibt die Umwelt auch wirklich zu schützen. Stattdessen gibt es weiterhin starke Anreize, sie zu übernutzen und zu belasten (perverse Anreizstruktur). Das heißt nicht, dass Umweltschutz nicht auch 
ohne oder gar gegen das ökonomische Kalkül - beispielweise aus ethisch-moralischen Motiven - entstehen kann. Es heißt vielmehr, dass die nötigen Verhaltensänderungen bei den Investitions- und Konsumentscheidungen unter den gegebenen Rahmenbedinungen nicht die Breite, Intensität und Dringlichkeit erreichen können, die wegen der inter-regionalen und inter-temporalen Dimension der Umweltproblematik eigentlich erforderlich wäre.

\section{$4 \quad$ Fazit}

Strukturwandel der Wirtschaft und Entlastung der Umwelt können theoretisch gesehen Hand in Hand gehen, doch in der Praxis ist Politik weiterhin gefragt - eine effektivere ökologische Politik allerdings, die vor allem - aber nicht nur - von zwei Ressorts formuliert und implementiert werden muss: der Umwelt- und der Wirtschaftspolitik.

Hier ist insbesondere an die weitere Definition von Nutzungsrechten und -pflichten, den verstärkten Einsatz marktorientierter Instrumente (wie Ökosteuern und Emissionszertifikate) und die Verschärfung des Umwelthaftungsrechts (Gefährdungshaftung) zu denken. Sobald Umweltbelastungen zu zusätzlichen einzelwirtschaftlichen Kosten führen, werden massive ökonomische Anreize freigesetzt, die die Innovationsfähigkeit des Marktmechanismus für einen ,ökologischen“S Strukturwandel aktivieren. Dabei ist die dynamische Effizienz umweltpolitischer Instrumente von besonderer Bedeutung.

Umweltauflagen (wie Grenzwerte und Standards) schreiben Vorgaben für die Zukunft fest die das gegenwärtige Wissen und den gegenwärtigen Stand der. Technik widerspiegeln. Wird ein Grenzwert erst einmal eingehalten, existiert kein weiterer Anreiz zur Einführung umweltentlastender Produkt- und Prozessinnovationen mehr. Dagegen stellen Ökosteuern und Emissionszertifikate permanente Anreize zur Innovation dar, denn jede Ressourcen- und Emissionsreduzierung führt ceteris paribus zu einer weiteren Senkung der Abgabenlast bzw. zu einer Erhöhung des unternehmerischen Handlungsspielraums. Auch haftungspolitische Maßnahmen, insbesondere die Gefährdungshaftung, können ein generelles Interesse an Innovationen wecken, den produktionsintegrierten Umweltschutz stärken und den intrasektoralen Strukturwandel in Richtung einer Umweltentlastung anregen und begünstigen.

Schrumpfung und Wachstum sind, wie die theoretische Reflexion zeigt inhärente Bestandteile des Strukturwandels der Wirtschaft, und sie sollten - so hat die empirische Analyse ergeben - zu elementaren Bestandteilen einer aktiven Strukturpolitik werden.

\section{Literatur}

Ayres, R.U., Udo E. Simonis (Hg.) (1994): Industrial Metabolism. Restructuring for Sustainable Development Tokyo/New York

BUND, Misereor (Hg.) (1996): Zukunftsfähiges Deutschland. Ein Beitrag zur nachhaltigen Entwicklung. Studie des Wuppertal Instituts, Basel/Berlin/Boston

Daly, Hermann E. (1994): Ökologische Ökonomie. Konzepte, Fragen, Folgerungen; in: JAHRBUCH ÖKOLOGIE 1995, München, 147-161

Deutsches Institut für Wirtschaftsforschung (1989): Stellungnahme des DIW zu den Fragen des Wirtschaftsausschusses des Deutschen Bundestages zum Thema „Entwicklung der ökologischen und sozialen Folgekosten des Wirtschaftens der Bundesrepublik Deutschland“, Manuskript Bonn 
Deutsches Institut für Wirtschaftsforschung (1988): Strukturverschiebungen zwischen sekundärem und tertiärem Sektor, Berlin: DIWBeiträge zur Strukturforschung

Grasskamp, R. u.a. (1992): Umweltschutz, Strukturwandel und Wirtschaftswachstum, Essen: RWI-Rheinisch-Westfälisches Institut für Wirtschaftsforschung

Härtel, H.-H. (1988): Wachstums- und Struktureffekte des Umweltschutzes, in: Wirtschaftsdienst 68, 245-252

Hinterberger, Friedrich, Fred Luks, Marcus Stewen (1996): Ökologische Wirtschaftspolitik. Zwischen Ökodiktatur und Umweltkatastrophe, Berlin/Basel/Boston

Huber, J. (1995): Nachhaltige Entwicklung. Strategien für eine ökologische und soziale Erdpolitik. Berlin

HWWA-Institut für Wirtschaftsforschung (1987): Zusammenhang zwischen Strukturwandel und Umwelt. Hamburg

Jänicke, Martin u.a. (1989): Structural Change and Environmental Impact. Empirical Evidence on Thirtyone Countries in East and West; in: Environmental Monitoring and Assessment, Vol. 12,1, 99-114

Jänicke, Martin u.a. (1993): Umweltentlastung durch industriellen Strukturwandel? Eine explorative Studie über 32 Industrieländer (1970-1990), Berlin

Junkernheinrich, M. (1993): Wirtschaftswachstum, Strukturwandel und Umweltschutz; in: Zeitschrift für angewandte Umweltforschung, 6.Jg.,4,412-418

Meadows, Dorella u.a. (1992): Die neuen Grenzen des Wachstums, Stuttgart

Sachverständigenrat für Umweltfragen (1996): Umweltgutachten 1996, Stuttgart

Schmidt-Bleek, Friedrich (1994): Wieviel Umwelt braucht der Mensch? MIPS - Das Maß für ökologisches Wirtschaften, Basel/Berlin/Boston

Sprenger, R.U., G. Britschkat (1981): Umweltschutz und sektoraler Strukturwandel, München

Umweltbundesamt (1997): Daten zur Umwelt 1996/97. Berlin

v. Weizsäcker, E.U., A.B. Lovins, H.L. Lovins (1995): Faktor Vier. Doppelter Wohlstand - halbierter Naturverbrauch. München

World Commission on Environment and Development (1987): Our Common Future, Oxford, New York

Weltkommission für Umwelt und Entwicklung (1987): Unsere gemeinsame Zukunft. Greven

Anschrift des Verfassers:

Prof. Dr. Udo E. Simonis

Wissenschaftszentrum Berlin für Sozialforschung

Umweltpolitik - Forschung

Reichpietschufer 50

D-10785 Berlin

\section{Schlagwörter: Nachhaltige Entwicklung, ökologische Politik, Strukturwandel}

